\title{
Research on Automatic Detection Method of Railway Fastener Defects Based on Image Processing
}

\author{
Qiu Yijin, Lv Zhaomin* \\ Email address: \\ 1435850697@qq.com (Qiu Yijin), zhaomin_lv@sues.edu.cn (Lv Zhaomin) \\ ${ }^{*}$ Corresponding author
}

School of Urban Rail Transportation, Shanghai University of Engineering Science, Shanghai, China

\section{To cite this article:}

Qiu Yijin, Lv Zhaomin. Research on Automatic Detection Method of Railway Fastener Defects Based on Image Processing. Science Discovery. Vol. 7, No. 6, 2019, pp. 429-435. doi: 10.11648/j.sd.20190706.19

Received: November 3, 2019; Accepted: December 9, 2019; Published: December 12, 2019

\begin{abstract}
With the rapid development of rail transit, the detection requirements for the various components of the track line are getting higher and higher, and relying on manual detection has the disadvantages of high cost and low efficiency. Therefore, it is urgent to study the method of automatic detection of track lines.This paper is based on the development history of computer vision and deep learning detection algorithm in fastener detection. It mainly introduces the related algorithms of positioning and classification, including the "cross" and template matching positioning algorithm; extracting the image direction gradient histogram The graph and the local binary pattern feature are merged, and the algorithm is classified by the support vector machine. At the same time, the convolutional neural network Alexnet architecture is used to extract the generalization characteristics of the fasteners to improve the classification accuracy of the fasteners. Finally, the problems and dilemmas of the existing fastener detection algorithms are discussed.
\end{abstract}

Keywords: Fastener Positioning, Fastener Classification, Deep Learning, Fastener Detect

\section{基于图像处理的铁路扣件缺陷自动检测方法的研究}

邱一晋, 吕照民*

上海工程技术大学城市轨道交通学院, 上海, 中国

\section{邮箱}

1435850697@qq.com (邱一晋), zhaomin_lv@sues.edu.cn（吕照民）

摘要: 随着轨道交通的高速发展, 对轨道线路各组成部分的检测要求也越来越高, 而依靠人工检测存在成本高, 效率低等 弊端, 因此研究能够实现轨道线路的自动化检测的方法成为了迫切的需求。本文基于计算机视觉和深度学习的检测算法在 扣件检测上应用的发展历程, 主要介绍了定位和分类两个环节的相关算法, 包括“十字交叉”和模板匹配的定位算法; 提取 图像方向梯度直方图和局部二值模式特征进行融合, 并用支持向量机进行分类的算法。同时, 比较采用卷积神经网络Alexnet 架构，提取扣件的泛化特征提高扣件的分类的精度，最后探讨现有扣件检测算法存在的问题与困境。

关键词: 扣件定位, 扣件分类, 深度学习, 扣件检测 


\section{1. 引言}

铁路基础设施的智能监控和检测是铁路提速发展的 必然要求，基于计算机视觉的检测技术是一种新型的铁路 安全检测技术，可以有效保证轨道交通工具的高效性和安 全性, 其中铁路扣件的自动巡检是一个重要组成部分。扣 件是连接钢轨和轨枕使之形成轨排的部件, 扣件的缺失可 能酿成列车脱轨等重大事故, 这对铁路的运营安全具有很 大的威胁。传统的铁路检测一直依靠人工和静态检测 [1], 这种检测缺乏实时性和准确性。近年来, 针对铁路扣件自 动化检测的研究, 国内外学者进行了积极的探索, 其中包 括了大量基于图像处理的扣件检测算法。

本文针对现有计算机视觉和深度学习在扣件检测领 域中的实际应用, 分别从扣件定位和状态分类两个环节, 对相关的铁路扣件的检测算法进行介绍和对比, 同时指出 现有算法面临的困境和存在的问题。

\section{2. 轨道扣件检测概述}

铁路扣件是将钢轨固定在轨枕上的紧固件, 扣件的丢 失、断裂很有可能会造成列车脱轨等重大事故[2], 实际线 路中每公里包含的铁路扣件多达7200个扣件, 因此实现轨 道扣件的自动化检测显得尤为重要[3-6]。

扣件的状态主要分为两种: 扣件异常, 和扣件正常, 其中扣件异常又包括扣件丢失、扣件松动和扣件断裂三种。 扣件的检测主要分为三个步骤：扣件图像预处理、扣件定 位、扣件状态分类。

由于原始采集到的轨道扣件图像特征信息不够明显, 以及受光照和拍摄仪器的影响会存在很多噪声, 所以图像 预处理是实现后续图像分割、特征提取、检测分类的前提, 是非常关键的一步, 它的效果直接影响到后续处理的准确 率和精度程度。在此涉及到的预处理包括: 基于高斯滤波 的图像去噪和基于直方图均衡化的图像增强。本文所介绍 的算法的详细流程如下图1所示:

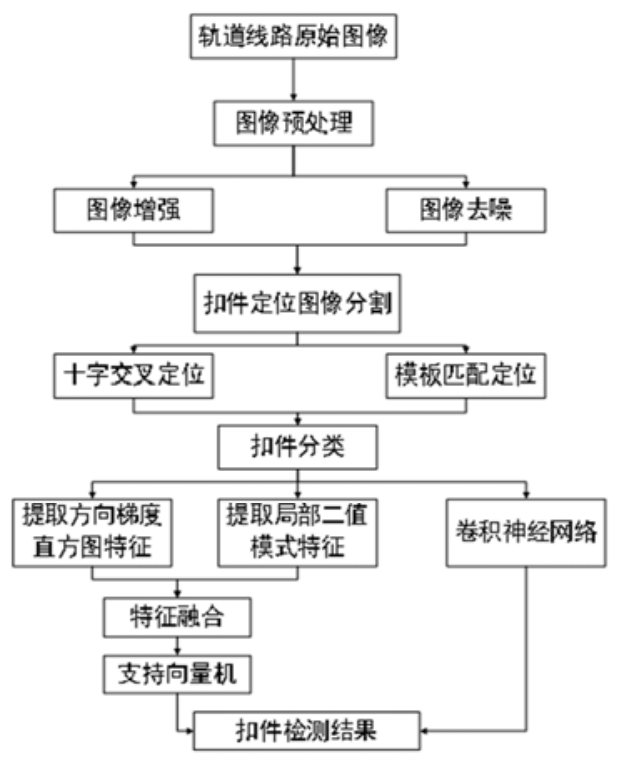

图1 检测算法流程图。

\section{3. 扣件区域定位分割}

对原始图像的扣件区域定位分割将有效缩小待检测 区域从而不仅减小了背景对扣件检测的影响, 同时由于缩 小了待检测区域也就提高了检测效率。目前常用的定位方 法有基于像素统计的定位方法和基于模板匹配的定位方 法。

像素统计的定位方法中最为人熟知的是“十字交叉” 定位方法,所谓“十字交叉”就是根据扣件图像的灰度值投 影规律找到能够在水平方向分割出钢轨区域的阈值, 以及 在坚直方向分割出轨枕区域的阈值，从而构成“十字”的交 叉形状最后依据先验知识定位扣件区域。其传统流程如下 图2所示。

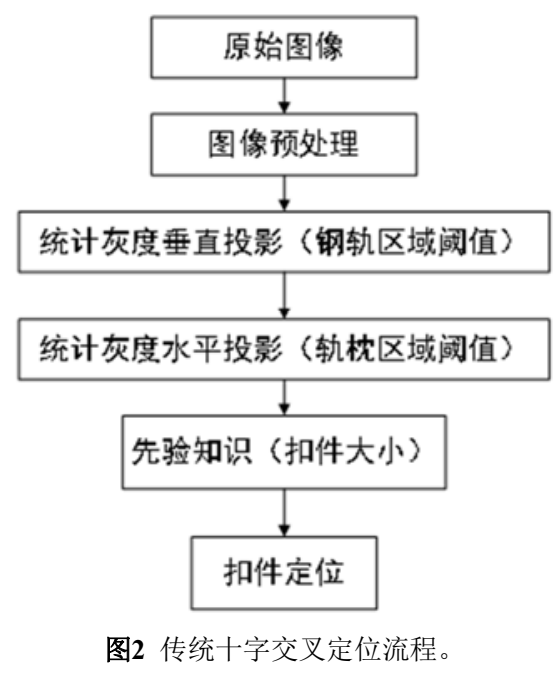

由上图可知, 对传统的十字交叉为了适应轨道线路的 复杂工况可以改进的地方有图像的预处理部分：文献[7] 中吴禄慎等研究了一种改进的十字交叉轨道扣件定位方 法, 提出通过先验知识缩小处理区域, 然后使用中值滤波 对图像预处理最后使用“十字交叉法”定位扣件; 钢轨和轨 枕区域定位部分由于统计的阈值仅能适应一条线路以及 存在人工统计找阈值的非自动化缺点, 文献[8]中提出一种 改进的Canny算子算法增强并提取出钢轨和轨枕的边缘, 再使用Hough变化提取直线特征的方法定位钢轨和轨枕区 域从而实现“十字交叉定位”。

模板匹配算法主要为模板图像在原图上进行由左往 右、由上到下进行相关运算, 最后得到一个进行相关运算 后的系数矩阵: 系数矩阵中的参数值用来度量模板图像在 原图中的子区域相似程度, 采用相关系数来进行评判。其 原理为将模板 $T(m, n)$ 叠放在搜索的原图 $S(W, H)$ 上, 进行滑动, 模板覆盖被搜索图的那块区域叫子图 $S_{i j}, i, j$ 为原图中子图所在坐标位置, 搜索范围为: $1 \leq i \leq W-n$, $1 \leq j \leq H-m$ 。可以用下式（1）衡量 $\mathrm{T}$ 和 $\mathrm{S}$ 的相似性:

$$
D(i, j)=\sum_{m=1}^{M} \sum_{n=1}^{N}\left[S^{i j}(m, n)-T(m, n)\right]^{2}
$$

其归一化的模板匹配的相关系数为: 


$$
R_{i j}=\frac{\sum_{m=1}^{M} \sum_{n=1}^{N} S^{i j}(m, n) \times T(m, n)}{\sqrt{\sum_{m=1}^{M} \sum_{n=1}^{N}\left[S^{i j}(m, n)\right]^{2}} \sqrt{\sum_{m=1}^{M} \sum_{n=1}^{N}[T(m, n)]^{2}}}(2)
$$

当模板图和子图一样时, 相关系数为 1 , 在被搜索图 完全搜索后, 找出 $R_{\text {max }}\left(i_{m}, j_{n}\right)$, 其对应子图即为匹配目标。 其流程如下图3所示。

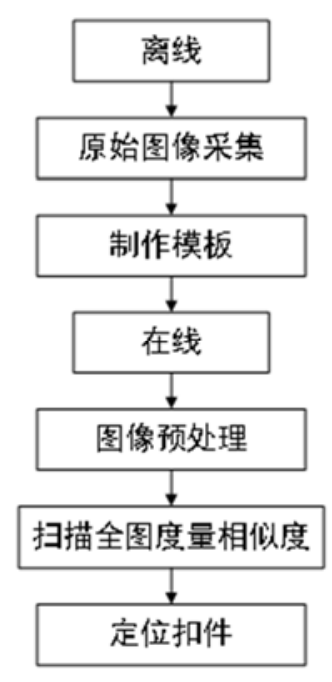

图3 模板匹配流程图。

由上图 3 可知, 为了适应铁路线路的各种工况, 针对 模板匹配定位算法的改进主要有对预处理方面的改进和 对相似性度量函数的改进, 范宏 [9]等提出一种改进的局部 二值模式 (Local Binary Pattern,LBP)来提取扣件的特征, 接着使用模板匹配的方法结合先验知识定位出了一幅图 片中的多个扣件, 且由于使用了LBP算法故能克服环境带 来的光照影响; 文献[10]则从度量相似度的角度出发选择 使用互信息来度量模板和原图间的相似度也取得了很好 的效果。

\section{4. 基于图像特征的扣件分类}

基于铁路高额的维修成本和巨大的工程量, 当对铁路 线路状态进行检测时, 采用图像处理的方法不仅能提高检 测效率，同时还能保证不对铁路线路造成破坏性检测，从 而可以做到不破坏铁路运营的完整性。在使用图像处理的 基础上本节提出首先提取图像方向梯度直方图(Histogram of Oriented Gridients,HOG)特征和LBP特征之后对特征进 行融合后使用支持向量机(Support Vector Machine,SVM) 进行分类器的训练和分类, 将扣件状态分为异常扣件和正 常扣件两类。

\section{1. 提取图像HOG特征}

HOG特征作为图像的一种形状特征是图像处理领域 的重要特征之一, 因其对图像光照和颜色的变化具有不敏 感性的特点, 被广泛应用于图像识别与检索等领域[11]。
提取图像的HOG特征时首先将图像划分成相同大小的 统计单元 [12], 将统计单元中各像素点的梯度方向或边缘方 向直方图的组合作为特征描述子。为了提高精确度, 可以 先计算各直方图在更大的图像区间中的密度, 并根据这个 密度值对各矢量单元做归一化, 以减少光照变化和阴影等 因素带来的影响, 获得更稳定的描述, 可以有效地描述物 体局部形状和表象。如图4即为HOG提取特征的流程图。

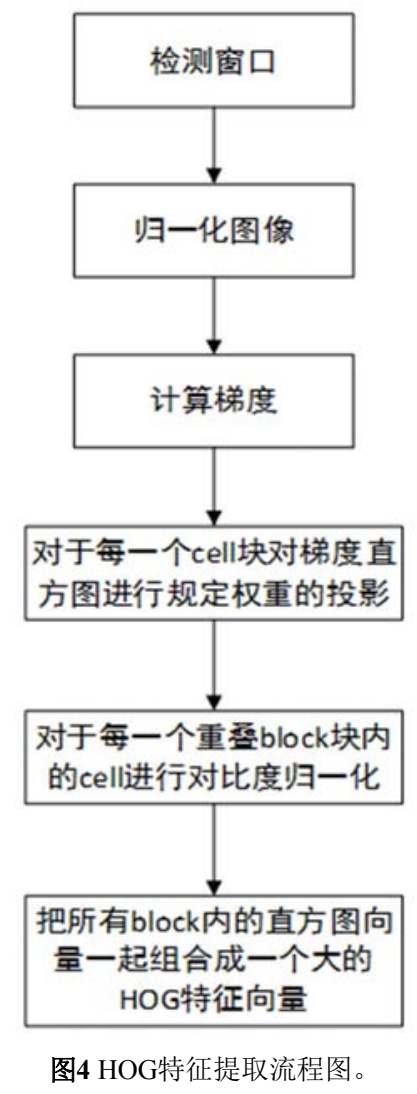

\section{2. 提取图像LBP特征}

LBP提取的特征是图像的局部纹理特征。由于其算法 简单, 运算效率高且具有灰度不变性等显著优点, 目前已 广泛运用于人脸识别和目标检测等领域。

对模拟图像可以采用二维离散函数 $f(x, y)$ 来表示, 其 中 $(x, y)$ 表示每个像素的位置坐标, $f(x, y)$ 则表示该坐标 处的像素值强度。原始的LBP算法采用 $3 \times 3$ 的邻域进行特 征提取, 即对于图像中的某一个像素 $P$ 设其坐标为 $(x, y)$, 则选取的 $3 \times 3$ 邻域其他像素坐标分别为 $(x+1, y)$, $(x+1, y-1),(x, y-1),(x-1, y-1), \quad(x-1, y)$, $(x-1, y+1),(x, y+1),(x+1, y+1)$ 。

该像素的特征表示方法, 首先, 通过一阶差分运算如 下式 (3) 计算得到该像素的邻域取值。

$$
\mathrm{g}_{i}=\left(p_{i}-p\right) / r, i=0,1,2 \ldots 7
$$

其中 $P_{i}, i=0,1,2 \ldots 7$, 按如图5顺序排列, $\mathrm{g}_{i}$ 表示一阶差 分运算取值, $\mathrm{r}$ 表示邻域半径。由于半径r对结果没有影响, 故忽略半径r, 只取差分部分结果 $p_{i}-p$ 。由于LBP算法计算 
的是邻域像素和中心像素的一阶梯度值, 而梯度在表达图像 特征时具有不受光照影响的特性, 这也就使得LBP算法在展 现图像纹理特征时具有较好的鲁棒性。之后定义如下判别式 (4) 得到该中心像素 8 邻域的二进制表达数值:

$$
P_{i}=\left\{\begin{array}{ll}
1 & x \geq 0 \\
0 & x<0
\end{array}, i=0,1,2 \ldots 7\right.
$$

其中 $x=p_{i}-p$ 。最后, 将该像素邻域的 8 个二进制数 按顺序组合, 并通过如下 (5) 式运算,便可以得到该像素 的LBP特征:

$$
\operatorname{LBP}(x, y)=\sum_{i=0}^{7} 2^{i} P_{7-i}
$$

其具体流程如图5所示。

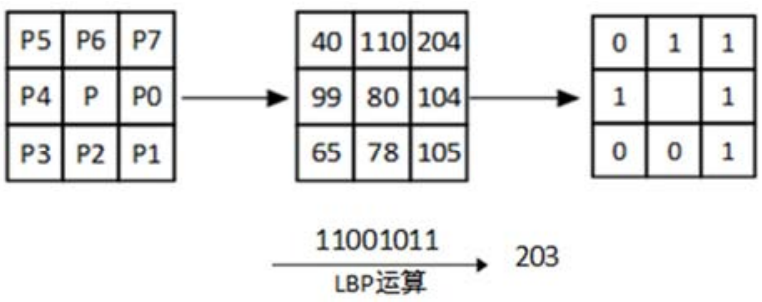

图5 原始的LBP原理。

经过上述对图像中每个像素提取LBP算子之后, 便 可以得到该图像的LBP特征图像，而LBP在应用于人脸 检测和纹理分类时, 如果直接用其特征图像进行识别 判断, 会由于位置关系不符合而导致误差, 因此在实 验时首先将一幅完整的图片进行划分, 再对划分后的 每一部分进行LBP特征提取, 最后在这个部分内统计 LBP特征的直方图。这样就可以用统计后的直方图来描 述每一个小的区域, 最后连接成整幅图像。

\section{3. 基于特征融合的扣件分类}

特征融合的方法主要有简单的级联融合和特征加 权融合的方式。考虑到特征加权需要进行大量的计算,
所以本文选用了简单的级联融合实现 HOG和LBP特征 的融合, 即把上述两个特征串行组合起来, 形成新的 特征, 该特征则不仅具有了图像的梯度特征还具有了 对光照环境的不敏感性。

支持向量机（Support Vector Machine,SVM）非常 适合解决非线性的小样本、高维空间识别问题。它的 特点是通过建立一个最优决策超平面, 使得尽可能让 超平面两侧的两类样本对象中距该平面最近点之间的 距离最大, 由此看出 SVM对于解决分类问题有很好的 适用性。

它的基本模型是一类线性分类器, 这类分类器的 特点是在特征空间上利用间隔最大化求最优分离超平 面 [13]。对于铁轨扣件的检测系统, 主要的分类为两类, 即正常的扣件和有异常的扣件, 将图库的图像融合后 的特征数据使用SVM进行训练, 在训练时依次把训练 集里面其中一个类别当成一个正类，把剩下的所有类 别当成一个负类。把测试样本的融合特征放到训练好 的SVM分类器模型中进行分类, 每一个测试样本都有 一个测试结果, 以此便可以分析算法的误报率和漏报 率。

\section{5. 基于深度学习的扣件分类}

基于图像处理的浅层机器学习通过提取“人工设 计特征”再搭配分类器进行图像分类虽然可以达到较 高的识别精度, 但是对图像的特征提取不够充分。而 且人工提取特征复杂, 且这种方法鲁棒性较差。

近年来, 随着深度学习的兴起, 深度学习在目标 检测和图像分类上以及成为热点。深度学习网络相比 于浅层机器学习可以提取更加丰富全面的图像特征, 将深度学习方法应用到轨道扣件缺陷检测也成为可能。 卷积神经网络对图像进行处理, 避免了复杂的手工提 取特征，特征提取更见丰富且与图像分类同时进行。 因此，本文将卷积神经网络Alexnet结构模型如下图6 所示, 应用于轨道扣件缺陷检测中, 并与前述的特征 融合后采用 SVM分类器分类的检测效果进行对比。

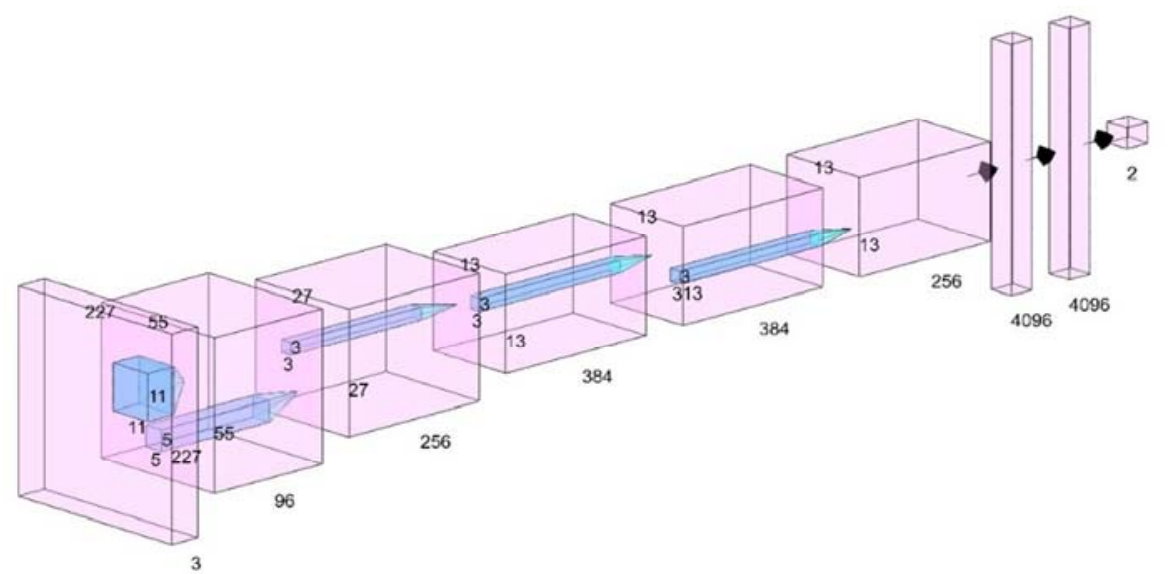

图6 Alexnet结构模型图。 


\section{1. 扣件图像处理}

对于训练Alexnet网络的图像进行处理。图像是具有 3 个通道的RGB图像, 将正常扣件标志为“1”, 异常扣件标准 为“ 0 ’。将图像调整为 $227 \times 227 \times 3$, 大小一致的图像更有 利于特征提取。

\subsection{Alexnet网络参数设置}

由于轨道扣件样本图片数量稀少, 而且重新训练一个网 络耗时长且精确度不高。因此采用迁移学习的技术, 冻结网 络的卷积层、池化层和激活函数, 将网络的后 3 个层给替换 成全连接层、分类层和输出层。采用使用随机梯度下降动量 优化器, 初始学习率为 0.0001 , 最多迭代的周期数 200 , 每个 周期迭代的批量大小为 10 。输出层大小根据输入图片的分类 多少决定, 为了在新层中比传输层更快的学习, 增加完全连 接层的权重学习率因子和偏置学习率因子的值为 10 。

\section{6. 实验结果分析}

本实验是基于Matlab、python、以及Halcon软件平台完 成的, 选用石家庄到太原的无砟线路进行了现场的扣件图片 采集, 钢轨扣件为弹条型扣件。选取 60 张图片作为样本其中 正样本 30 张, 异常样本 30 张, 测试图片 22 张, 其中正样本 11 张, 负样本 11 张。采用主频为 $2.4 \mathrm{GHz}$ 的计算机机型仿真实验。

\section{1. 扣件定位结果分析}

如下图7所示即为十字交叉定位的扣件效果图。图中 竖直的蓝色矩形框是根据灰度垂直投影的确定的钢轨区 域阈值所得到的, 而水平的蓝色矩形框所框选出的是根据 灰度水平投影所确定的扣件区域阈值得到的, 结合扣件大 小这一先验知识, 两者呈十字交叉框选出了扣件区域。

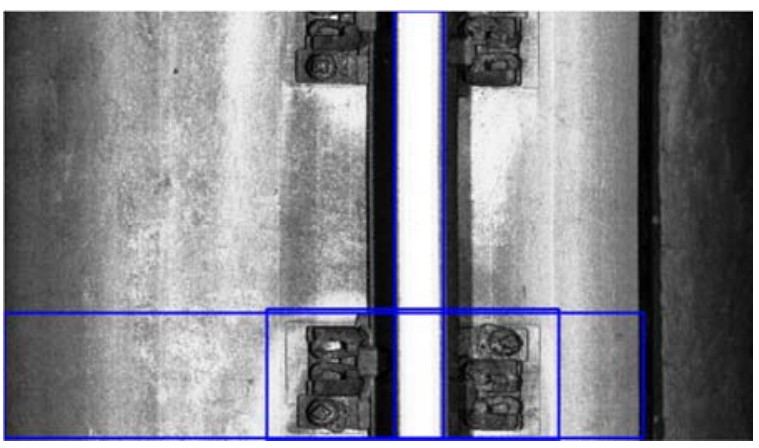

图7 十字交叉的扣件定位图。

如下图8所示即为模板匹配的效果图。其中图(a)为所 用模板图片 (一个正常的有砟线路钢轨右侧扣件)，图(b) 为无砟线路图经灰度增强后用模板匹配的方法定位到扣 件图, 图中红色矩形框框选区域即为扣件区域。

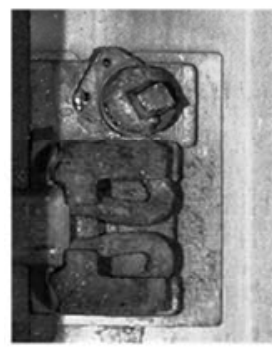

(a)

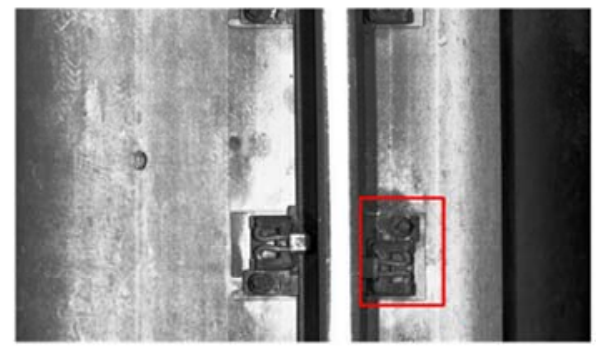

(b)

图8 模板匹配结果图。

\section{2. 扣件分类结果分析}

$\mathrm{HOG}$ 提取后的特征图如下图图9所示。

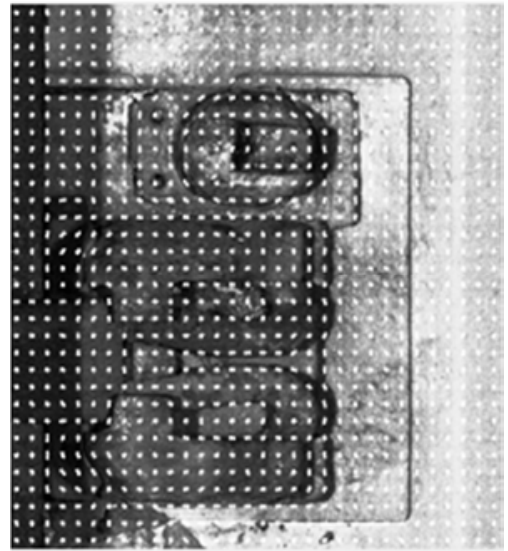

图9 HOG提取的扣件特征图。
LBP提取后的特征图如下图10所示。

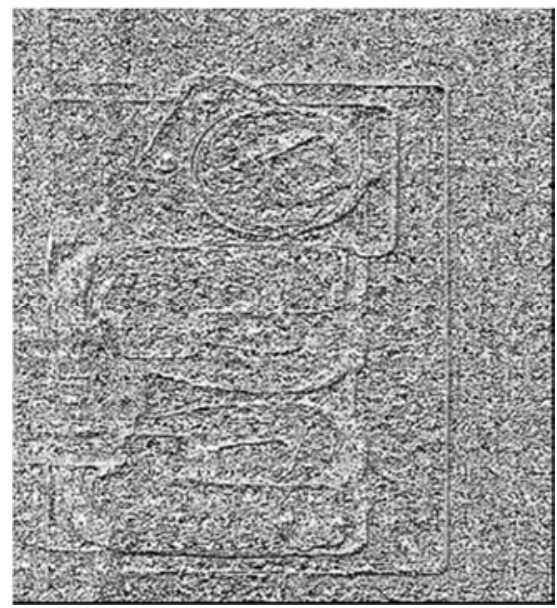

图10 LBP提取的扣件特征图。 
通过特征融合和SVM分类得出的扣件结果展示如下 图11所示。其中图(a)为异常扣件中发生断裂的扣件，图(b)
为被识别出的正常扣件。

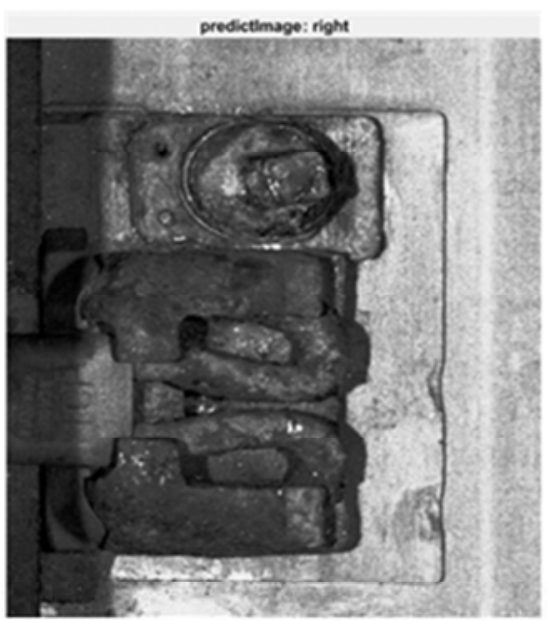

(b)

图11 扣件状态识别效果图。

通过实验计算了本文所提到的算法的扣件检测效果 的评价指标用下表所示的误报率、漏报率和准确率进行描 述, 准确率越高, 误报率及漏报率越低则扣件的分类检测 效果越好。

表1 扣件检测分类实验结果。

\begin{tabular}{llll}
\hline 算法 & 误报率(\%) & 漏报率(\%) & 准确率(\%) \\
\hline HOG+SVM & 20 & 0 & 90 \\
HOG+LBP+SVM & 18 & 0 & 91 \\
Alexnet深度学习 & 10.9 & 0 & 94.56 \\
\hline
\end{tabular}

通过上述分析HOG和LBP特征融合后用 SVM的方法 可以有效识别出扣件状态其准确率达到了 $91 \%$, 且漏报率 为 0 因而可以用于线路的检测中, 而Alexnet网络搭建的深 度学习的方法其各方面较之特征融合和svm组合的方法则 更好些其准确率达到了 $94.56 \%$ 。

\section{7. 结论}

由本文的实验和证明发现了现有扣件检测技术存在 的问题与困境如下：1）扣件定位算法在直线段的使用已 经较为成熟, 但在道岔路段的使用研究成果较少；2）现 有的扣件检测技术在对扣件异常的研究展开的较少; 3) 扣件的异常样本数据量少, 这对于依赖大样本的深度学习 算法和利用图像特征的算法存在很大的限制；4）现有的 扣件分类识别算法在面对复杂背景的线路图时为了提高 准确率, 采用了融合特征的办法, 但也只能在一定程度上 提高准确率。为了解决这些问题, 提出使用多种特征融合 的办法提高检测的准确率, 并使用无监督学习的办法降低 算法对异常样本大量需求的限制, 课题组将在此后对该方 向进行深入研究。

\section{致谢}

本文为上海市科学技术委员会纵向项目 《Shanghai Sailing Program》（18YF1409200）的阶段性成果之一。

\section{参考文献}

[1] 王强,李柏林,侯云,范宏.一种改进的LBP特征实现铁路扣件 识别[J].西南交通大学学报,2018,53(05):893-899.

[2] 王开雄,何彪,李柏林, 等.结合掩膜和可变形部件模型的扣 件定位算法 [J].计算机工程与应用,2019.

[3] 罗建桥,刘甲甲,李柏林，等.基于局部特征和语义信息的扣 件图像检测 [J]. 计算机应用研究,2016,33(8):2514-2518,2523.

[4] Zhang H B, Yang J F, Tao W, et al. Vision method of inspecting missing fastening components in highspeed railway [J]. Applied Optics, 2011, 50 (20): 3658-3665.

[5] Yang Jinfeng, Tao Wei, Liu Manhua, et al. An efficient direction field-based method for the detection of fasteners on high-speedrailways [J]. Sensors, 2011, 11 (8): 7364 -7381.

[6] Marino F, Distante A, Mazzeo P L, et al. A real-time visual inspection system for railway maintenance:automatic hexagonal-headed bolts detection [J]. IEEE Transactions on Systems, Man, and Cybernetics, Part C, 2007, 37 (3): 418-428.

[7] 吴禄慎, 万超, 陈华伟, 等. 一种改进的十字交叉轨道扣件 定位方法[J]. 铁道标准设计, 2016(12):49-53.

[8] 邹逸,顾桂梅,张军平. 基于改进Canny算子的铁路扣件定位 方法[J].兰州交通大学学报,2019,38(01):72-77+94.

[9] 范宏,侯云,李柏林,熊鹰.高铁扣件的自适应视觉检测算法 [J/OL].西南交通大学学报:1-6[2019-11-01]. 
[10] 谢凤英,吴叶芬,周世新.基于互信息的铁路轨枕扣件自动定 位算法[J].中国体视学与图像分析,2013,18(02):145-149.

[11] 代国忠.图像识别技术在铁轨扣件异常检测中的应用研究 [D].哈尔滨工程大学, 2018 .
[12] 李永波,李柏林,熊鹰.基于 $\mathrm{HOG}$ 特征的铁路扣件状态检测 [J]. 传感器与微系统,2013,32(10):110-113.

[13] 韩金岳. 基于多特征融合的轨道螺栓扣件图像识别技术研 究[D].兰州交通大学, 2018 .

\section{作者简介}

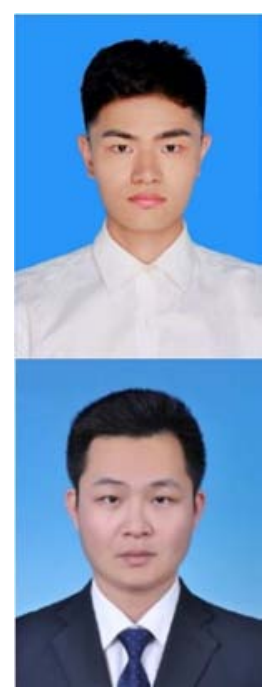

邱一晋 1996年7月出生于江苏省, 上海工程技术大学, 城市轨道交通学院专业硕士, 主要从事轨 道交通智能检测方面的研究。

吕照民 1990年1月出生于内蒙古, 2012年本科毕业于华东理工大学自动化专业, 2017年博士毕业 于华东理工大学控制科学与工程专业, 研究方向为轨道交通智能检测, 化工过程。 